\title{
Écologie des Stomoxes (Diptera : Muscidae) au Gabon. II. ORIGINE DES REPAS DE SANG ET CONSÉQUENCES ÉPIDÉMIOLOGIQUES
}

\author{
MAVOUNGOU J.F.*****, SIMO G.**, GILLES J.***, DE STORDEUR E.**** \& DUVALLET G.*****
}

Summary: ECology of STOMOXYINE Flies (Diptera: Muscidae) IN GABON. II. BLOOD MEALS ANALYSIS AND EPIDEMIOLOGIC CONSEQUENCES To determine the origin of stomoxyine fly bloodmeals (Diptera: Muscidae) in Gabon, 1,021 flies belonging to seven different species of Stomoxys were captured and dissected in the area of Makokou. In total, 798 were not blood-fed and 223 bloodmeals could be gathered on filter paper. The identification of the origin of these meals was made by amplification of mitochondrial Cytb gene, then heteroduplex technique by using the Gambian rat (Cricetomys gambianus) as driver. Samples of fauna, collected on the local market, consisted of 24 mammal and two reptile blood and muscle samples, to which it is necessary to add human samples (27 potential hosts). 19 meals could not be amplified for technical reasons, 65 were amplified, but the acquired patterns corresponded to none of the tested potential hosts. On the 139 identified meals, $55 \%$ were taken on the black-fronted duiker (Cephalophus nigrifrons) and $19 \%$ on pig. Stomoxys transvittatus, the most abundant species in Makokou, is very opportunistic: 68 $\%$ of meals were taken on six different hosts, among whom $48 \%$ on the black-fronted duiker and $32 \%$ were not identified using the panel of tested hosts. S. xanthomelas took $50 \%$ of its meals on the moustached monkey (Cercopithecus cephus) and $7 \%$ on human beings. S. calcitrans, species of anthropised areas, took $33 \%$ of its meals on human beings. These three species can therefore take bloodmeals on wild fauna and human beings. They could potentially play an important role in the emergence of zoonotic diseases. The four other species took their bloodmeals only on wild fauna and pig, the only example of domestic fauna in this study. This preliminary study must be followed up using a larger number of specimens and by increasing the diversity of the tested potential hosts.

KEY WORDS : Stomoxys spp., ecology, epidemiology, bloodmeals, PCR, heteroduplex, Gabon.

I es stomoxes (Diptera: Muscidae) sont des mouches hématophages, de 3 à $10 \mathrm{~mm}$ de longueur, qui ont l'aspect général d'une mouche domestique (Musca domestica Linné, 1758) avec des pièces bucca-

\footnotetext{
* Institut de Recherche en Écologie Tropicale (IRET), BP 13354, Libreville, Gabon.

** Centre de Recherches Médicales, Institut de Recherche Médicale et d'Études sur les plantes Médicinales (IMPM), Yaoundé, Cameroun. **:* Institut für Vergleichende Tropenmedizin und Parasitologie, München, Allemagne.

*:***: UMR 5175 CEFE (Centre d'Écologie Fonctionnelle et Évolutive), Université de Montpellier Languedoc-Roussillon, Montpellier, France. Correspondance : Jacques F. Mavoungou, UMR 5175 (CEFE), Université Paul Valéry, F-34199 Montpellier Cedex 5, France.

E-Mail : mavoungoujacques@yahoo.fr
}

\section{Résumé :}

Pour déterminer l'origine des repas de sang des stomoxes (Diptera: Muscidae) au Gabon, 1021 mouches appartenant à sept espèces différentes ont été capturées et disséquées dans la région de Makokou. Au total, 798 n'étaient pas gorgées et 223 repas de sang ont pu être recueillis sur papier filtre. L'identification de l'origine de ces repas a été faite par amplification du gène mitochondrial Cytb puis formation d'hétéroduplex en utilisant le rat de Gambie comme driver. Les prélèvements de faune, sur le marché local, comprenaient 24 mammifères et deux reptiles, auxquels il faut ajouter l'homme, soit 27 hôtes potentiels. 19 repas n'ont pu être amplifiés pour des raisons techniques; 65 ont été amplifiés, mais les patterns obtenus ne correspondaient à aucun des profils des hôtes potentiels testés. Sur les 139 repas identifiés, 55 \% ont été pris sur le Céphalophe à front noir (Cephalophus nigrifrons) et $19 \%$ sur le porc. Stomoxys transvittatus, espèce la plus abondante à Makokou, est très opportuniste : $68 \%$ des repas ont été pris sur six hôtes différents, dont $48 \%$ sur le céphalophe à front noir, et $32 \%$ n'ont pas été identifiés sur les hôtes testés. S. xanthomelas a pris $50 \%$ de ses repas sur singe Moustac (Cercopithecus cephus) et $7 \%$ sur homme. S. calcitrans, espèce des milieux anthropisés, a pris $33 \%$ de ses repas sur homme. Ces trois espèces peuvent donc prendre leurs repas de sang sur la faune sauvage et sur homme. Elles pourraient ainsi jover un rôle important dans l'émergence de zoonoses. Les quatre autres espèces n'ont pris leurs repas que sur la faune sauvage et le porc, seul exemple d'animal domestique dans cette étude. Cette étude préliminaire doit être poursuivie sur un plus grand nombre d'individus, en augmentant la diversité des hôtes potentiels testés.

MOTS CLÉS : Stomoxys spp., écologie, épidémiologie, repas de sang, PCR, hétéroduplex, Gabon.

les piqueuses. Ces insectes sont un fléau pour la faune domestique ou sauvage et parfois l'homme (Zumpt, 1973) en raison de leur nuisance directe (harcèlement dû à la piqûre et prédation sanguine), et de leur rôle vecteur potentiel de divers agents pathogènes (Leclercq, 1971; Zumpt, 1973; Mihok et al., 1995; Foil, 1989; Foil \& Gorham, 2000; Mramba et al., 2007). Ce groupe est connu essentiellement à travers une espèce cosmopolite, Stomoxys calcitrans (L., 1758). A Makokou (Gabon), sept espèces de stomoxes ont été identifiées (Mavoungou et al., 2008). Leur biologie demeure largement inconnue. À la différence des glossines et des moustiques où une masse importante d'informations sur l'origine de repas de sang est disponible (Moloo, 1993; Ngumbi 
et al., 1992; Boakye et al., 1999; Torr et al., 2001; Njiokou et al., 2004), les hôtes de plusieurs espèces de stomoxes sont inconnus, ou demeurent encore peu documentés (Kangwagye, 1977; Mihok et al., 1996). Or la détermination de l'origine des repas sanguins des insectes vecteurs peut aider à déterminer les hôtes potentiels et les pathogènes pouvant être transmis. De plus, la connaissance détaillée des comportements alimentaires d'un insecte sur les différents hôtes vertébrés est considérée comme un pré-requis pour la mise en place d'une stratégie efficace de lutte contre les éventuelles épidémies. Compte tenu du caractère opportuniste de certaines espèces de Diptères hématophages, comme Glossina palpalis palpalis (Laveissière et al., 1985) par exemple, l'identification du repas sanguin est très importante parce qu'elle donne des indications sur les réservoirs animaux des pathogènes et sur les facteurs entomologiques qui pourraient contribuer à la transmission de ces pathogènes.

Le but de cette étude a été de déterminer l'origine des repas de sang des différentes espèces de stomoxes capturées à Makokou dans le nord-est du Gabon. C'est une étape indispensable pour une meilleure compréhension de leur rôle épidémiologique dans la transmission de pathogènes.

\section{MATÉRIEL ET MÉTHODES}

T es stomoxes ont été collectés à Makokou $\left(00^{\circ}\right.$ $30.701^{\prime} \mathrm{N}$ et $12^{\circ} 48.1524^{\prime} \mathrm{E}$ ) dans le nord-est du $\checkmark$ Gabon. Les caractéristiques de cette zone ont été décrites ailleurs (Vande Weghe, 2006). Ces insectes ont été capturés à l'aide du piège Vavoua (Laveissière \& Grébaut, 1990) pendant le mois d'août 2006. Ils ont été ensuite disséqués et les repas de sang contenus dans leurs tubes digestifs ont été collectés sur des disques de papier Wattman $\mathrm{n}^{\circ} 2\left(2 \mathrm{~cm}^{2}\right)$, et stockés dans des tubes Eppendorf ${ }^{\circledR}$.

Pour avoir une bonne identification des repas de sang, il était nécessaire d'avoir des échantillons de la faune vertébrée locale. Des échantillons de sang et de muscles ont donc été collectés sur la faune sauvage auprès des vendeuses de gibier sur le marché de Makokou et auprès des chasseurs (tableau I). Les gouttes de sang des animaux prélevés ont été déposées sur des disques de papier Wattman $\mathrm{n}^{\circ} 2\left(2 \mathrm{~cm}^{2}\right)$ et les échantillons de muscles ont été conservés dans des tubes Eppendorf ${ }^{\circledR}$ contenant de l'éthanol à $95^{\circ}$ jusqu'à extraction de l'ADN.

\section{EXTRACTION DE L'ADN DES TISSUS ANIMAUX}

L'ADN a été extrait à partir de $25 \mathrm{mg}$ de tissu en utilisant le kit "DNeasy Blood \& Tissue" (Qiagen ${ }^{\circledR}$ ). $400 \mu 1$ d'éluat contenant l'ADN ont été récupérés.

\begin{tabular}{ll}
\hline \multicolumn{1}{c}{ Noms communs } & \multicolumn{1}{c}{ Noms scientifiques } \\
\hline Mone à pieds noir & Cercopithecus mona pogonias \\
Moustac & Cercopithecus cephus \\
Cercocèbe à joues grises & Lophocebus albigena \\
Talapoin & Miopithecus ogouensis, \\
Céphalophe à bandes dorsales & Cephalophus dorsalis \\
noires & \\
Céphalophe bleu & Cephalophus monticola \\
Céphalophe à front noir & Cercopithecus nigrifrons \\
Sitatunga & Tragelaphus spekei gratus \\
Chevrotin aquatique & Hyemoschus aquaticus \\
Mangouste brune & Crossarchus obscurus \\
Mangouste à pattes noires & Bdeogale nigripes \\
Aulacode & Thryonomys swinderianus \\
Rat de Gambie & Cricetomys gambianus \\
Nandinie & Nandinia binotata \\
Athérure & Atherurus africanus \\
Chat doré & Felis aurata \\
Pangolin à écailles tricuspides & Manis tricuspis \\
Pangolin à longue queue & Manis longicaudata \\
Pangolin géant & Manis gigantea \\
Potto de Bosman & Perodictitus potto \\
Potto de calabar & Arctocebus calabarensis \\
Écureuil à pieds rouges & Heliosciurus rufobrachium \\
Potamochère & Potamochoerus porcus \\
Faux gavial & Crocodylus cataphractus \\
Varan du Nil & Varanus niloticus \\
Porc & Sus scrofa domestica \\
Homme & Homo sapiens \\
\hline &
\end{tabular}

Tableau I. - Noms communs et scientifiques des vertébrés constituant les échantillons de faune pour l'étude de l'origine des repas de sang des stomoxes.

\section{EXTRACTION DE L'ADN DES REPAS DE SANG}

À chaque repas de sang contenu dans un tube Eppendorf $^{\circledR}, 1 \mathrm{ml}$ de chelex $(5 \%)$ a été ajouté. Les tubes ont été incubés dans un bain-marie à $56^{\circ} \mathrm{C}$ pendant une heure puis à $100^{\circ} \mathrm{C}$ pendant 30 minutes. À la suite d'une centrifugation à $15000 \mathrm{~g}$ pendant 10 minutes, le surnageant contenant l'ADN a été récupéré pour être utilisé directement pour la $\mathrm{PCR}$, ou stocké à $-20^{\circ} \mathrm{C}$.

\section{IDENTIFICATION DES REPAS DE SANG}

L'identification a été faite selon la technique basée sur l'amplification du gène mitochondrial du cytochrome $\mathrm{B}$, suivie de la formation des hétéroduplex entre les produits d'amplification du Rat de Gambie et ceux des autres vertébrés ou des repas de sang (Njiokou et al., 2004)

\section{AmplificATION DU GENE DU CYTOCHROME B}

La réaction d'amplification a été faite dans $25 \mu \mathrm{l}$ de mélange contenant $5 \mu \mathrm{l}$ de solution d'ADN, $10 \mathrm{mM}$ de Tris-HCl (pH 9), $50 \mathrm{mM}$ de $\mathrm{KCl}, 1,5 \mathrm{mM}$ de $\mathrm{MgCl}_{2}$, 20 picomoles de chaque amorce, $200 \mu \mathrm{M}$ de chaque dNTP (désoxynucléotide 5'-triphosphate) et 0,5 unité de Taq DNA polymérase (Appligene-Oncor, USA). Une dénaturation à $95^{\circ} \mathrm{C}$ pendant 3,5 minutes a précédé 40 cycles d'amplification. Chaque cycle comprenait une 


\begin{tabular}{|c|c|c|c|c|c|c|c|c|c|}
\hline \multirow[b]{2}{*}{ Sources de repas de sang } & \multicolumn{9}{|c|}{ Espèces de stomoxes } \\
\hline & S. $t$. & S. $x$. & S. $o$. & S. $i$. & S. n. n. & S. $n . b$. & S. $c$. & $N D$ & Total \\
\hline Chat doré & 2 & 1 & - & - & - & - & - & - & 3 \\
\hline Moustac & 2 & 7 & 1 & 2 & 2 & - & - & - & 14 \\
\hline Céphalophe à front noir & 57 & 1 & 4 & 12 & - & 1 & 1 & 1 & 77 \\
\hline Porc & 11 & 1 & 6 & - & 3 & - & - & 1 & 22 \\
\hline Pangolin géant & 8 & - & - & - & 5 & - & - & - & 13 \\
\hline Mangouste & - & - & - & - & - & - & 1 & - & 1 \\
\hline Homme & 2 & 1 & - & - & - & - & 6 & - & 9 \\
\hline Repas amplifiés et non identifiés & 38 & 3 & 11 & 3 & - & - & 10 & - & 65 \\
\hline Sous total & 120 & 14 & 22 & 17 & 10 & 1 & 18 & 2 & 204 \\
\hline Repas non amplifié & 5 & 1 & 1 & 2 & 0 & 0 & 10 & 0 & 19 \\
\hline Total & 125 & 15 & 23 & 19 & 10 & 1 & 28 & 2 & 223 \\
\hline
\end{tabular}

S.t. = Stomoxys tranvittatus, S. x. = S. xanthomelas, S. o. = S. omega, S. i. = S. inornatus, S.n.n. = S. niger niger, S.n. b. = S. niger bilineatus, S. c. $=$ S. calcitrans.

$\mathrm{ND}=$ individus non déterminés.

Tableau II. - Identification de l'origine des repas de sang de différentes espèces de stomoxes à Makokou (Gabon) par la technique des profils hétéroduplex.

dénaturation à $95^{\circ} \mathrm{C}$ pendant 30 secondes, une hybridation des amorces à $58^{\circ} \mathrm{C}$ pendant 50 secondes et enfin une élongation à $72^{\circ} \mathrm{C}$ pendant 40 secondes. Une élongation finale a été réalisée à $72^{\circ} \mathrm{C}$ pendant cinq minutes. L'amplification du gène du cytochrome $\mathrm{B}$ a été vérifiée par électrophorèse sur gel d'agarose contenant du bromure d'éthidium $(0,5 \mu \mathrm{g} / \mu \mathrm{l})$.

\section{FORMATION ET ANALYSE DES HÉTÉRODUPLEX}

Les hétéroduplex ont été formés en ajoutant, dans un microtube de $0,5 \mathrm{ml}, 8 \mu \mathrm{l}$ de tampon TE (Tris-EDTA), $4 \mu \mathrm{l}$ de chaque produit d'amplification, et un volume égal du produit d'amplification du "driver" (Rat de Gambie ou Cricetomys gambianus). Pour éviter l'évaporation, deux gouttes d'huile minérale ont été ajoutées au contenu de chaque microtube. Après une dénaturation des produits d'amplification par incubation du mélange à $98^{\circ} \mathrm{C}$ pendant 2,5 minutes, un refroidissement lent (plus de 30 minutes) à la température ambiante a permis de former les hétéroduplex. Au contenu de chaque microtube, $6 \mu \mathrm{l}$ du tampon de l'échantillon $(0,25 \%$ bleu de bromophénol, 0,25\% xylène cyanol et $30 \%$ glycérol) ont été ensuite ajoutés, et l'ensemble a été séparé sur gel d'acrylamide (5\%) contenant de l'urée (1 $\mathrm{M}$ en concentration finale). L'électrophorèse a été réalisée pendant 18 heures sous $12 \mathrm{~mA}$ et $83 \mathrm{~V}$. Le gel a été coloré au bromure d'éthidium $(0,5 \mu \mathrm{g} / \mathrm{ml})$, lavé, puis visualisé sous UV et enfin photographié.

\section{RÉSULTATS}

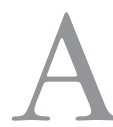

u total 1021 stomoxes ont été disséqués et 223 repas de sang recueillis ( 798 de ces mouches n'étaient donc pas gorgées). 204 repas de sang ont été amplifiés et l'origine de 139 repas a pu être identifiée par la formation de profils hétéroduplex et comparaison aux profils obtenus avec la faune locale. La synthèse des résultats est présentée dans le tableau II. L'origine de 65 repas n'a pu être identifiée car leurs profils hétéroduplex ne correspondaient à aucun profil formé par l'ADN des hôtes de référence; 19 repas n'ont pas été amplifiés.

Sur les 139 repas identifiés, $55 \%$ ont été pris sur le Céphalophe à front noir (Cephalophus nigrifrons Gray, 1871), et $19 \%$ sur le porc. À noter que pour l'espèce S. xanthomelas $50 \%$ des repas amplifiés ont été pris sur le singe Moustac (Cercopithecus cephus), pour l'espèce $S$. omega $50 \%$ des repas amplifiés n'ont pu être identifiés avec la gamme d'hôtes à notre disposition, et pour l'espèce $S$. calcitrans $33 \%$ des repas amplifiés ont été pris sur homme.

\section{DISCUSSION}

Ce travail réalisé à proximité de Makokou (Gabon) est la première tentative d'identification de l'origine des repas de sang des différentes espèces de stomoxes (Diptera : Muscidae) capturées dans cette région d'Afrique équatoriale.

Chez les stomoxes, les deux sexes sont hématophages, mais l'on sait que ces insectes peuvent aussi survivre en absorbant du nectar (Mihok et al., 1996). Un repas de sang est cependant obligatoire pour le développement des oufs et la ponte chez les femelles. Cela pourrait expliquer qu'une majorité de mouches capturées (798 sur 1021) ne contenait pas de sang dans leur tube digestif. Une partie d'entre elles également pouvait être des mouches ténérales, venant d'émerger, et qui n'avait pas pris leur premier repas sanguin. 19 repas n'ont pas pu être amplifiés pour des raisons techniques; 65 ont 
été amplifiés mais n'ont pu être identifiés, leurs profils hétéroduplex ne correspondant à aucun des profils formés avec l'ADN des hôtes de référence à notre disposition. Dans ce travail préliminaire, nous n'avons pu disposer de prélèvements venant de la faune domestique (bovins, ovins, chiens, volailles) à l'exception du porc; de même, aucune espèce d'oiseaux sauvages ne figure dans la liste d'hôtes identifiables.

Si globalement $55 \%$ des repas de sang amplifiés ont été pris sur le céphalophe à front noir, c'est le fait essentiellement de deux espèces $S$. transvittatus et $S$. inornatus. La première, qui est l'espèce la plus abondante dans cette zone (Mavoungou et al., 2008), semble la plus opportuniste en prenant des repas de sang sur six des sept hôtes identifiés. Malgré un échantillon faible (15) pour l'espèce $S$. xanthomelas, on constate que $50 \%$ de ses repas de sang ont été pris sur le singe moustac. Cela est à mettre en relation avec l'observation que nous avons faite de l'inféodation probable de cette espèce à la canopée (Mavoungou et al., 2007). Cette préférence trophique pour une espèce de singe arboricole confirme que la niche écologique de cette espèce de stomoxes est sans doute la canopée. Un des repas de sang de cette espèce ayant été pris sur homme, on observe l'importance épidémiologique que pourrait avoir celle-ci en faisant le lien entre les populations simiesques et humaines. Il faut noter aussi que l'espèce opportuniste $S$. transvittatus a pris des repas de sang à la fois sur le singe moustac et sur l'homme. L'espèce S. calcitrans a pris $33 \%$ de ses repas amplifiés sur l'homme. Cela confirme son caractère anthropophile (Zumpt, 1973; Campbell et al., 1977; Wieman et al., 1992; Catangui et al., 1997; Campbell et al., 1987; Mavoungou et al., 2008) qui fait que cette espèce se rencontre essentiellement dans des milieux anthropisés et qu'elle soit devenue cosmopolite en suivant l'homme dans ses pérégrinations à travers le monde. Plus de $55 \%$ des repas amplifiés de cette espèce n'ont pu être identifiés. Cela tient vraisemblablement à l'absence de faune domestique, à l'exception du porc, dans les hôtes testés.

Notre étude montre que la majorité des repas identifiés ont été pris sur le Céphalophe à front noir ( C. nigrifrons). Ces résultats rejoignent ceux obtenus par Mihok et al. (1996), qui montraient que certaines espèces de stomoxes au Kenya prennent la majorité de leurs repas de sang sur une autre antilope, le Guib harnaché (Tragelaphus scriptus Pallas). Le porc apparaît aussi dans notre étude comme hôte des stomoxes. Les stomoxes ont été considérés depuis longtemps comme vecteurs mécaniques de trypanosomes (D'Amico et al., 1996). Nos observations semblent confirmer ce lien vectoriel que les stomoxes pourraient établir entre faune sauvage, réservoir potentiel de trypanosomes, et faune domestique. De même, deux espèces, $S$. transvittatus et $S$. xanthomelas, qui peuvent prendre des repas de sang à la fois sur singe et sur homme, pourraient jouer un rôle épidémiologique important dans la transmission de plusieurs pathogènes, en particulier des virus. La technique des hétéroduplex a permis d'identifier facilement l'origine des repas de sang des stomoxes. Pour les repas amplifiés mais non identifiés, certains profils hétéroduplex ne correspondent pas à ceux des espèces animales utilisées comme référence. À partir de là, il sera possible d'intégrer ou de rechercher d'autres espèces potentiellement hôtes des stomoxes.

Cette étude est tout à fait préliminaire, car le nombre d'hôtes potentiels devant être pris en compte doit être augmenté pour élargir l'échantillon à la faune domestique et aux oiseaux. Elle montre néanmoins que ce groupe négligé de diptères hématophages devrait être pris en compte dans de futures études épidémiologiques.

\section{REMERCIEMENTS}

\begin{abstract}
e travail a été réalisé en partie grâce à un financement de l'Union européenne via le Projet Fonds Européen de Développement 8 ACP GA 009 (PSVAP - Composante II Makokou, CNPN-MESRIT-U.E./ IRET-CIFOR). Il a été réalisé en partie également dans le cadre du projet ANR-IFORA (Les îles forestières africaines : modèle d'une nouvelle approche de la dynamique de structuration de la biodiversité). Nous tenons à remercier Philippe Hecketsweiller, Paul Posso, Robert Nasi, Charles Doumenge, Gérard Cuny, Thierry Baldet, Stéphane Herder, Rosalie Ngoua, Joseph Okouyi, Nicolas Yao Emba, pour leur aide technique.
\end{abstract}

\section{RÉFÉRENCES}

Boakye D.A., Tang J., Truc P., Merriweather A. \& Unnasch T.R. Identification of bloodmeals in haematophagous Diptera by cytochrome B heteroduplex analysis. Medical and Veterinary Entomology, 1999, 13, 282-287.

Campbell J.B., Berry I.L., Boxler D.J., Davis R.L., Clanton D.C. \& Deutscher G.H. Effects of stable flies (Diptera: Muscidae) on weight gain and feed efficiency of feedlot cattle. Journal of Economic Entomology, 1987, 80, 117-119.

Campbell J.B., White R.G., Wright J.E., Crookshank R. \& Clanton D.C. Effects of stable flies (Diptera-Muscidae) on weight gains and feed-efficiency of calves on growing and finishing rations. Journal of Economic Entomology, 1977, 70, 592-594.

Catangui M.A., Campbell J.B., Thomas G.D. \& Boxler D.J. Calculating economic injury levels for stable flies (Diptera: Muscidae) on feeder heifers. Journal of Economic Entomo$\log$, 1997, 90, 6.

D'Amico F., Gouteux J.P., Le Gall F. \& Cuisance D. Are stable flies (Diptera: Stomoxyinae) vectors of Trypanosoma vivax in the Central African Republic? Veterinary Research, 1996, 27, 161-170. 
Foll L.D. Tabanids as vectors of disease agents. Parasitology today, 1989, 5, 88-96.

Foll L.D. \& GORHAM J.R. Mechanical transmission of disease agents by arthropods, in: Medical Entomology, Eldridge B.F. \& Edman J.D. (Eds). Kluwer Academic Publishers, Dordrecht, The Netherlands, 2000, 461-514

KANGWAGYE T.N. Reactions of large mammals to biting flies in Ruwenzori National Park, Uganda. Advances in Medical, Veterinary and Agricultural Entomology Eastern Africa (ed. by C.P.F. de Lima), East African Literature Bureau, Nairobi, $1977,32-44$.

Lavessière C., Couret D., StaAk C. \& Hervouet J.P. Glossina palpalis et ses hôtes en secteur forestier de Côte d'Ivoire. Relation avec l'épidémiologie de la trypanosomiase humaine. Cahier ORSTOM, Série Entomologie Médicale et Parasitologie, 1985, 23, 297-303.

LAvessière C. \& GréBaut P. The trapping of tsetse flies (Diptera: Glossinidae). Improvement of a model: the Vavoua trap. Tropical Medicine and Parasitology, 1990, 41, 185-192.

LECLERCQ M. Les mouches nuisibles aux animaux domestiques. Presses agronomiques de Gembloux, 1971.

Mavoungou J.F., Gilles J. \& Duvallet G. Stomoxys xanthomelas Roubaud, 1937 : une espèce de la canopée en Afrique équatoriale? (Diptera : Muscidae). Bulletin de la Société entomologique de France, 2007, 112, 481-483.

Mavoungou J.F., Jay-Robert P., Gilles J., Atsame Edda A. \& Duvallet G. Écologie des stomoxes (Diptera: Muscidae) au Gabon. I. Premier inventaire dans différentes zones écologiques. Parasite, 2008, 15, 27-34.

Mihok S., Maramba O., Munyoki E., \& Kagoiya J. Mechanical transmission of Trypanosoma spp. by African Stomoxyinae. Tropical Medecine and Parasitology, 1995, 46, 103-105.

Minok S. \& Clausen P.H. Feeding habits of Stomoxys spp. stable flies in a Kenyan forest. Medical and Veterinary Entomology, 1996, 10, 392-394.

Moloo S.K. The distribution of Glossina species in Africa and their natural hosts. Insect Science and its Application, $1993,14,511-527$.

Mramba F., Broce A. B. \& Zurek L. Vector competence of stable flies, Stomoxys calcitrans L. (Diptera: Muscidae), for Enterobacter sakazakii. Journal of vector Ecology, 2007, 32, 134-139.

Ngumbi P.M., Lawyer P.G., Johson R.N., Kilu G. \& Asiago C. Identification of phlebotomine sandfly bloodmeals from Baringo district, Kenya, by direct enzyme-linked immunosorbent-assay (ELISA). Medical and Veterinary Entomology, 1992, 6, 385-388.

Nitokou F., Simo G., Mbida Mbida A., Truc P., Cuny G. \& Herder S. A study of preference in tsetse flies using a modified heteroduplex PCR-based method. Acta Tropica, 2004, 91, 117-120.

Torr S.J., Wilson P.J., Schofield S., Mangwiro T.N., Akber S., \& White B.N. Application of DNA markers to identify the individual-specific hosts of tsetse feeding on cattle. Medical and Veterinary Entomology, 2001, 15, 78-86.

VAnde Weghe J.P. Les parcs nationaux du Gabon. Ivindo \& Mwagna. Eaux noires, forêts vierges et baïs. Wildlife Conservation Society, Libreville, Gabon, 2006, 272 p.
Wieman G.A., Campbell J.B., Deshazer J.A. \& Berry I.L. Effects of stable flies (Diptera: Muscidae) and Heat-stress on Weight-Gain and Feed-Efficiency of Feeder Cattle. Journal of Economic Entomology, 1992, 85, 1835-1842.

Zumpt F. The Stomoxyine Biting Flies of the World (Diptera: Muscidae). Taxonomy, biology, economic importance and control measures. Stuttgart: Gustav Fisher Verlag, 1973, 175 p.

Reçu le 17 janvier 2008 Accepté le 19 mars 2008 